\title{
A hybrid projection method for solving a common solution of a system of equilibrium problems and fixed point problems for asymptotically strict pseudocontractions in the intermediate sense in Hilbert spaces
}

Chatchawan Watchararuangwit ${ }^{1}$, Pongrus Phuangphoo ${ }^{1,2}$ and Poom Kumam ${ }^{1 *}$

"Correspondence:

poom.kum@kmutt.ac.th

'Department of Mathematics,

Faculty of Science, King Mongkut's University of Technology Thonburi

(KMUTT), Bang Mod, Thung Kru, Bangkok, 10140, Thailand

Full list of author information is available at the end of the article

\begin{abstract}
In this paper, we introduce a new iterative algorithm which is constructed by using the hybrid projection method for finding a common solution of a system of equilibrium problems of bifunctions satisfying certain conditions and a common solution of fixed point problems of a family of uniformly Lipschitz continuous and asymptotically $\lambda_{i}$-strict pseudocontractive mappings in the intermediate sense. We prove the strong convergence theorem for a new iterative algorithm under some mild conditions in Hilbert spaces. Finally, we also give a numerical example which supports our results.

MSC: $47 \mathrm{H} 05 ; 47 \mathrm{H} 09 ; 47 \mathrm{H} 10$
\end{abstract}

Keywords: asymptotically strict pseudocontraction in the intermediate sense; hybrid projection method; system of equilibrium problems; fixed point problems

\section{Introduction}

Let $C$ be a closed and convex subset of a real Hilbert space $H$ with the inner product $\langle\cdot, \cdot\rangle$ and the norm $\|\cdot\|$. Let $\left\{F_{m}\right\}_{m \in \Gamma}$ be a family of bifunctions from $C \times C$ into $\mathbb{R}$, where $\mathbb{R}$ is the set of real numbers and $\Gamma$ is an arbitrary index set. The system of equilibrium problems is to find $x \in C$ such that

$$
F_{m}(x, y) \geq 0, \quad m \in \Gamma, \forall y \in C .
$$

The set of solutions of (1.1) is denoted by $\operatorname{SEP}\left(F_{m}\right)$, where $m \in \Gamma$, that is,

$$
\operatorname{SEP}\left(F_{m}\right)=\left\{x \in C: F_{m}(x, y) \geq 0, \forall y \in C\right\} .
$$

If $\Gamma$ is a singleton, then the problem (1.1) is reduced to the equilibrium problem of finding $x \in C$ such that

$$
F(x, y) \geq 0, \quad \forall y \in C .
$$

@ 2012 Watchararuangwit et al.; licensee Springer. This is an Open Access article distributed under the terms of the Creative Commons Attribution License (http://creativecommons.org/licenses/by/2.0), which permits unrestricted use, distribution, and reproduction in any medium, provided the original work is properly cited. 
The set of solutions of (1.3) is denoted by $\mathrm{EP}(F)$.

Recall the following definitions.

A mapping $A: C \rightarrow H$ is called monotone if

$$
\langle A x-A y, x-y\rangle \geq 0, \quad \forall x, y \in C .
$$

A mapping $A$ is called $\alpha$-inverse-strongly monotone $[1,2]$, if there exists a positive real number $\alpha$ such that

$$
\langle A x-A y, x-y\rangle \geq \alpha\|A x-A y\|^{2}, \quad \forall x, y \in C .
$$

Clearly, if $A$ is $\alpha$-inverse-strongly monotone, then $A$ is monotone.

A mapping $A$ is called $\beta$-strongly monotone if there exists a positive real number $\beta$ such that

$$
\langle A x-A y, x-y\rangle \geq \beta\|x-y\|^{2}, \quad \forall x, y \in C .
$$

A mapping $A$ is called $L$-Lipschitz continuous if there exists a positive real number $L$ such that

$$
\|A x-A y\| \leq L\|x-y\|, \quad \forall x, y \in C .
$$

It is easy to see that if $A$ is an $\alpha$-inverse-strongly monotone mapping from $C$ into $H$, then $A$ is $\frac{1}{\alpha}$-Lipschitz continuous.

In 2009, Qin et al. [3] introduced the following algorithm for a finite family of asymptotically $\lambda_{i}$-strictly pseudocontractions.

Let $x_{0} \in C$ and $\left\{\alpha_{n}\right\}_{n=0}^{\infty}$ be a sequence in $(0,1)$. The sequence $\left\{x_{n}\right\}$ is as follows:

$$
\left\{\begin{array}{l}
x_{1}=\alpha_{0} x_{0}+\left(1-\alpha_{0}\right) S_{1} x_{0}, \\
x_{2}=\alpha_{1} x_{1}+\left(1-\alpha_{1}\right) S_{2} x_{1}, \\
x_{3}=\alpha_{2} x_{2}+\left(1-\alpha_{2}\right) S_{3} x_{2}, \\
\vdots \\
x_{N}=\alpha_{N-1} x_{N-1}+\left(1-\alpha_{N-1}\right) S_{N} x_{N-1}, \\
x_{N+1}=\alpha_{N} x_{N}+\left(1-\alpha_{N}\right) S_{1}^{2} x_{N}, \\
x_{N+2}=\alpha_{N+1} x_{N+1}+\left(1-\alpha_{N+1}\right) S_{2}^{2} x_{N+1}, \\
\vdots \\
x_{2 N}=\alpha_{2 N-1} x_{2 N-1}+\left(1-\alpha_{2 N-1}\right) S_{N}^{2} x_{2 N-1}, \\
x_{2 N+1}=\alpha_{2 N} x_{2 N}+\left(1-\alpha_{2 N}\right) S_{1}^{3} x_{2 N}, \\
\vdots
\end{array}\right.
$$

It is called the explicit iterative sequence of a finite family of asymptotically $\lambda_{i}$-strictly pseudocontractions $\left\{S_{1}, S_{2}, \ldots, S_{N}\right\}$. Since for each $n \geq 1$, it can be written as $n=(h-1) N+i$, 
where $i=i(n) \in\{1,2,3, \ldots, N\}, h=h(n) \geq 1$ is a positive integer and $h(n) \rightarrow \infty$, as $n \rightarrow \infty$, we can rewrite the above table in the following compact form:

$$
x_{n}=\alpha_{n-1} x_{n-1}+\left(1-\alpha_{n-1}\right) S_{i(n)}^{h(n)} x_{x-1}, \quad \forall n \geq 1 .
$$

Next, Sahu et al. [4] introduced new iterative schemes for asymptotically strictly pseudocontractive mappings in the intermediate sense. To be more precise, they proved the following theorem.

Theorem (SXY) Let $C$ be a nonempty closed and convex subset of a real Hilbert space $H$ and $T: C \rightarrow C$ be a uniformly continuous asymptotically $\kappa$-strictly pseudocontractive mapping in the intermediate sense with a sequence $\left\{\gamma_{n}\right\}$ such that $F(T)$ is nonempty and bounded. Let $\left\{\alpha_{n}\right\}$ be a sequence in $[0,1]$ such that $0<\delta \leq \alpha_{n} \leq 1-\kappa$ for all $n \in \mathbb{N}$. Let $\left\{x_{n}\right\} \subset C$ be a sequence generated by the following (CQ) algorithm:

$$
\left\{\begin{array}{l}
u=x_{1} \in C \text { chosen arbitrarily, } \\
y_{n}=\left(1-\alpha_{n}\right) x_{n}+\alpha_{n} T^{n} x_{n}, \\
C_{n}=\left\{z \in C:\left\|y_{n}-z\right\|^{2} \leq\left\|x_{n}-z\right\|^{2}+\theta_{n}\right\}, \\
Q_{n}=\left\{z \in C:\left\langle x_{n}-z, u-x_{n}\right\rangle \geq 0\right\}, \\
x_{n+1}=P_{C_{n} \cap Q_{n}}(u), \quad \forall n \in \mathbb{N},
\end{array}\right.
$$

where $\theta_{n}=c_{n}+\gamma_{n} \Delta_{n}$ and $\Delta_{n}=\sup \left\{\left\|x_{n}-z\right\|: z \in F(T)\right\}<\infty$. Then $\left\{x_{n}\right\}$ converges strongly to $P_{F(T)}(u)$, where $P_{F(T)}$ is a metric projection from $H$ into $F(T)$.

In 2010, $\mathrm{Hu}$ and Cai [5] considered the asymptotically strictly pseudocontractive mappings in the intermediate sense concerning the equilibrium problem. They obtained the following result in a real Hilbert space. Next, Ceng et al. [6] introduced the viscosity approximation method for a modified Mann iteration process for asymptotically strict pseudocontractive mappings in the intermediate sense and they proved the strong convergence of a general CQ-algorithm and extended the concept of asymptotically strictly pseudocontractive mappings in the intermediate sense to the Banach space setting called nearly asymptotically strictly pseudocontractive mappings in the intermediate sense. Finally, they established a weak convergence theorem for a fixed point of nearly asymptotically strictly pseudocontractive mappings in the intermediate sense which are not necessarily Lipschitz continuous mappings.

Theorem (HC) Let $C$ be a nonempty closed and convex subset of a real Hilbert space $H$ and $N \geq 1$ be an integer, $\phi: C \rightarrow C$ be a bifunction satisfying (A1)-(A4), and $A: C \rightarrow H$ be an $\alpha$-inverse-strongly monotone mapping. Let for each $1 \leq i \leq N, T_{i}: C \rightarrow C$ be a uniformly continuous $k_{i}$-strictly asymptotically pseudocontractive mapping in the intermediate sense for some $0 \leq k_{i}<1$ with sequences $\left\{\gamma_{n, i}\right\} \subset[0, \infty)$ such that $\lim _{n \rightarrow \infty} \gamma_{n, i}=0$ and $\left\{c_{n, i}\right\} \subset$ $[0, \infty)$ such that $\lim _{n \rightarrow \infty} c_{n, i}=0$. Let $k=\max \left\{k_{i}: 1 \leq i \leq N\right\}, \gamma_{n}=\max \left\{\gamma_{n, i}: 1 \leq i \leq N\right\}$, and $c_{n}=\max \left\{c_{n, i}: 1 \leq i \leq N\right\}$. Assume that $\digamma=\bigcap_{i=1}^{N} F\left(T_{i}\right) \cap \mathrm{EP}(\phi)$ is nonempty and bounded. Let $\left\{\alpha_{n}\right\}$ and $\left\{\beta_{n}\right\}$ be sequences in $[0,1]$ such that $0<a \leq \alpha_{n} \leq 1,0<\delta \leq \beta_{n} \leq 1-k$ for all $n \in \mathbb{N}$, and $0<b \leq r_{n} \leq c<2 \alpha$. 
Let $\left\{x_{n}\right\}$ and $\left\{u_{n}\right\}$ be sequences generated by the following algorithm:

$$
\left\{\begin{array}{l}
x_{1} \in C \text { chosen arbitrarily, } \\
u_{n} \in C \text { such that } \phi\left(u_{n}, y\right)+\left\langle A x_{n}, y-u_{n}\right\rangle+\frac{1}{r_{n}}\left\langle y-u_{n}, u_{n}-x_{n}\right\rangle \geq 0, \\
\quad \forall y \in C, \\
z_{n}=\left(1-\beta_{n}\right) u_{n}+\beta_{n} T_{i(n)}^{h(n)} u_{n}, \\
y_{n}=\left(1-\alpha_{n}\right) u_{n}+\alpha_{n} z_{n}, \\
C_{n}=\left\{v \in C:\left\|y_{n}-v\right\|^{2} \leq\left\|x_{n}-v\right\|^{2}+\theta_{n}\right\}, \\
Q_{n}=\left\{v \in C:\left\langle x_{n}-v, x_{0}-x_{n}\right\rangle \geq 0\right\}, \\
x_{n+1}=P_{C_{n} \cap Q_{n}}\left(x_{0}\right), \quad \forall n \in \mathbb{N} \cup\{0\},
\end{array}\right.
$$

where $\theta_{n}=c_{h(n)}+\gamma_{h(n)} \rho_{n}^{2} \rightarrow 0$, as $n \rightarrow \infty$, and $\rho_{n}=\sup \left\{\left\|x_{n}-v\right\|: v \in \digamma\right\}<\infty$. Then $\left\{x_{n}\right\}$ converges strongly to $P_{\digamma}\left(x_{0}\right)$.

In 2011, Duan and Zhao [7] introduced new iterative schemes for finding a common solution set of a system of equilibrium problems and a solution of a fixed point set of asymptotically strict pseudocontractions in the intermediate sense and they proved these schemes converge strongly.

In 2012, Shui Ge [8] introduced a new hybrid algorithm with variable coefficients for a fixed point problem of a uniformly Lipschitz continuous mapping and asymptotically pseudocontractive mapping in the intermediate sense on unbounded domains and he proved strong convergence in a real Hilbert space.

Theorem (Ge) Let $C$ be a nonempty, closed, and convex subset of a real Hilbert space $H$, $T: C \rightarrow C$ be a uniformly L-Lipschitz continuous mapping and asymptotically pseudocontractive mapping in the intermediate sense with sequences $\left\{k_{n}\right\} \subset[1, \infty)$ and $\left\{v_{n}\right\} \subset[0, \infty)$. Let $q_{n}=2 k_{n}-1$ for each $n \in \mathbb{N}$. Let $\left\{x_{n}\right\}$ be the sequence generated by the following hybrid algorithm with variable coefficients:

$$
\left\{\begin{aligned}
x_{1} \in & C \text { chosen arbitrarily, } \\
C_{1}= & C, \\
z_{n}= & \left(1-\hat{\beta}_{n}\right) x_{n}+\hat{\beta}_{n} T^{n} x_{n}, \\
y_{n}= & \left(1-\hat{\alpha}_{n}\right) x_{n}+\hat{\alpha}_{n} T^{n} z_{n}, \\
C_{n}= & \left\{u \in C_{n}:\left\|y_{n}-u\right\|^{2} \leq\left\|x_{n}-u\right\|^{2}\right. \\
& \left.-\hat{\alpha}_{n} \hat{\beta}_{n}\left(1-\hat{\beta}_{n}-\hat{\beta}_{n}^{2} L^{2}-q_{n} \hat{\beta}_{n}\right)\left\|x_{n}-T^{n} x_{n}\right\|^{2}+\alpha_{n} \theta_{n}\right\}, \\
x_{n+1}= & P_{C_{n+1}}\left(x_{1}\right), \quad \forall n \in \mathbb{N},
\end{aligned}\right.
$$

where

$$
\begin{aligned}
& \theta_{n}=2\left(1+r_{0}^{2}\right)\left(q_{n}-1\right)\left(1+q_{n} \hat{\beta}_{n}\right)+2\left(1+q_{n} \hat{\beta}_{n}\right) v_{n} \\
& \hat{\alpha}_{n}=\frac{\alpha_{n}}{1+\left\|x_{n}-x_{1}\right\|^{2}} \quad \text { and } \quad \hat{\beta}_{n}=\frac{\beta_{n}}{1+\left\|x_{n}-x_{1}\right\|^{2}} .
\end{aligned}
$$


Assume that the positive real number $r_{0}$ is chosen so that $B_{r_{0}}\left(x_{1}\right) \cap \operatorname{Fix}(T) \neq \emptyset$ and that $\left\{\alpha_{n}\right\}$ and $\left\{\beta_{n}\right\}$ are sequences in $(0,1)$ such that $a \leq \alpha_{n} \leq \beta_{n} \leq b$ for some $a>0$ and for some $b \in\left(0, \frac{1}{2+L}\right)$.

Then $\left\{x_{n}\right\}$ converges strongly to a fixed point of $T$.

In this paper, motivated and inspired by the previously mentioned above results, we introduce a new iterative algorithm by the hybrid projection method for finding a common solution of a system of equilibrium problems of bifunctions satisfying certain conditions and a common solution of fixed point problems of a family of uniformly Lipschitz continuous and asymptotically $\lambda_{i}$-strict pseudocontractive mappings in the intermediate sense in a real Hilbert space. Then, we prove a strong convergence theorem of the iterative algorithm generated by this conditions. Finally, we also give a numerical example which supports our results. The results obtained in this paper extend and improve several recent results in this area.

\section{Preliminaries}

Let $H$ be a real Hilbert space with the inner product $\langle\cdot, \cdot\rangle$ and the norm $\|\cdot\|$. Let $C$ be a closed and convex subset of $H$. For any point $x \in H$, there exists a unique nearest point in $C$, denoted by $P_{C}(x)$, such that

$$
\left\|x-P_{C} x\right\| \leq\|x-y\|, \quad \forall y \in C .
$$

$P_{C}$ is called the metric projection of $H$ onto $C$ defined by the following:

$$
P_{C}(x)=\arg \min _{y \in C}\|x-y\| .
$$

We know that $P_{C}$ is a nonexpansive mapping $H$ onto $C$. It is also known that $P_{C}$ satisfies

$$
\left\|P_{C} x-P_{C} y\right\|^{2} \leq\left\langle P_{C} x-P_{C} y, x-y\right\rangle, \quad \forall x, y \in H
$$

and

$$
\left\langle x-P_{C} x, z-P_{C} x\right\rangle \geq 0, \quad \forall z \in C .
$$

We will adopt the following notations:

(1) $\rightarrow$ for strong convergence and $\rightarrow$ for weak convergence.

(2) $\omega_{w}\left(x_{n}\right)=\left\{x: \exists x_{n_{j}} \rightarrow x\right\}$ denotes the weak $w$-limit set of $\left\{x_{n}\right\}$.

(3) A nonlinear mapping $S: C \rightarrow C$ is a self-mapping in $C$. We denote the set of fixed points of $S$ by $F(S)$ (i.e., $F(S)=\{x \in C: S x=x\}$ ). Recall the following definitions.

Definition 2.1 Let $S$ be a mapping from $C$ to $C$. Then

(1) $S$ is said to be nonexpansive if

$$
\|S x-S y\| \leq\|x-y\|, \quad \forall x, y \in C .
$$

(2) $S$ is said to be uniformly Lipschitz continuous if there exists a constant $L>0$ such that

$$
\left\|S^{n} x-S^{n} y\right\| \leq L\|x-y\|, \quad \text { for all integers } n \geq 1, \forall x, y \in C \text {. }
$$


(3) $S$ is said to be asymptotically nonexpansive if there exists a sequence $\left\{k_{n}\right\} \subset[1, \infty)$ with $k_{n} \rightarrow 1$ as $n \rightarrow \infty$ such that

$$
\left\|S^{n} x-S^{n} y\right\| \leq k_{n}\|x-y\|, \quad \text { for all integers } n \geq 1, \forall x, y \in C .
$$

The class of asymptotically nonexpansive mappings was introduced by Goebel and Kirk (see [9]) in 1972. It is known that if $C$ is a nonempty, bounded, closed, and convex subset of a real Hilbert space $H$, then every asymptotically nonexpansive self-mapping has a fixed point. Further, the set $F(S)$ of fixed points of $S$ is closed and convex.

(4) $S$ is said to be asymptotically nonexpansive in the intermediate sense $[10,11]$ if it is continuous and the following inequality holds:

$$
\limsup _{n \rightarrow \infty} \sup _{x, y \in C}\left(\left\|S^{n} x-S^{n} y\right\|-\|x-y\|\right) \leq 0 .
$$

Putting $\xi_{n}=\max \left\{0, \sup _{x, y \in C}\left(\left\|S^{n} x-S^{n} y\right\|-\|x-y\|\right)\right\}$, we see that $\xi_{n} \rightarrow 0$ as $n \rightarrow \infty$. Then (2.4) is reduced to

$$
\left\|S^{n} x-S^{n} y\right\| \leq\|x-y\|+\xi_{n}, \quad \forall x, y \in C .
$$

The class of asymptotically nonexpansive mappings in the intermediate sense was introduced by Kirk and Bruck et al. (see $[10,11]$ ) as a generalization of the class of asymptotically nonexpansive mappings. It is known that if $C$ is a nonempty, bounded, closed, and convex subset of a real Hilbert space $H$, then every asymptotically nonexpansive self-mapping in the intermediate sense has a fixed point (see [12]).

(5) $S$ is said to be contractive if there exists a coefficient $k \in(0,1)$ such that

$$
\|S x-S y\| \leq k\|x-y\|, \quad \forall x, y \in C .
$$

(6) $S$ is said to be a $\lambda$-strict pseudocontraction if there exists a coefficient $\lambda \in[0,1)$ such that

$$
\|S x-S y\|^{2} \leq\|x-y\|^{2}+\lambda\|(I-S) x-(I-S) y\|^{2}, \quad \forall x, y \in C .
$$

The class of strict pseudocontractions was introduced by Brower and Petryshyn (see [1]) in 1967. Clearly, if $S$ is a nonexpansive mapping, then $S$ is a strict pseudocontraction with $\lambda=0$. We also remark that if $\lambda=1$, then $S$ is called a pseudocontractive mapping.

(7) $S$ is said to be an asymptotically $\lambda$-strict pseudocontraction with the sequence $\left\{d_{n}\right\}$ (see also [13]) if there exists a sequence $\left\{d_{n}\right\} \subset[0, \infty)$ with $d_{n} \rightarrow 0$ as $n \rightarrow \infty$ and a constant $\lambda \in[0,1)$ such that

$$
\begin{aligned}
& \left\|S^{n} x-S^{n} y\right\|^{2} \leq\left(1+d_{n}\right)\|x-y\|^{2}+\lambda\left\|\left(x-S^{n} x\right)-\left(y-S^{n} y\right)\right\|^{2}, \\
& \forall x, y \in C, \forall n \in \mathbb{N} .
\end{aligned}
$$

The class of asymptotically strict pseudocontractions was introduced by Qihou [14] in 1996. Clearly, if $S$ is an asymptotically nonexpansive mapping, then $S$ is an asymptotically 
strict pseudocontraction with $\lambda=0$. We also remark that if $\lambda=1$, then $S$ is said to be an asymptotically pseudocontractive mapping which was introduced by Schu [15] in 1991.

(8) $S$ is said to be an asymptotically $\lambda$-strict pseudocontraction in the intermediate sense with the sequence $\left\{d_{n}\right\}[4,5]$ if there exists a sequence $\left\{d_{n}\right\} \subset[0, \infty)$ with $d_{n} \rightarrow 0$ as $n \rightarrow \infty$ and a constant $\lambda \in[0,1)$ such that

$$
\begin{aligned}
& \limsup _{n \rightarrow \infty} \sup _{x, y \in C}\left(\left\|S^{n} x-S^{n} y\right\|^{2}-\left(1+d_{n}\right)\|x-y\|^{2}-\lambda\left\|\left(x-s^{n} x\right)-\left(y-s^{n} y\right)\right\|^{2}\right) \leq 0, \\
& \forall x, y \in C, \forall n \in \mathbb{N} .
\end{aligned}
$$

Putting $c_{n}=\max \left\{0, \sup _{x, y \in C}\left(\left\|S^{n} x-S^{n} y\right\|^{2}-\left(1+d_{n}\right)\|x-y\|^{2}-\lambda\left\|\left(x-s^{n} x\right)-\left(y-s^{n} y\right)\right\|^{2}\right)\right\}$, we see that $c_{n} \rightarrow 0$ as $n \rightarrow \infty$. Then (2.8) is reduced to

$$
\left\|S^{n} x-S^{n} y\right\|^{2} \leq\left(1+d_{n}\right)\|x-y\|^{2}+\lambda\left\|\left(x-s^{n} x\right)-\left(y-s^{n} y\right)\right\|^{2}+c_{n}, \quad \forall x, y \in C, \forall n \in \mathbb{N} .
$$

The class of asymptotically strict pseudocontractions in the intermediate sense was introduced by Sahu, $\mathrm{Xu}$, and Yao [4] as a generalization of a class of asymptotically strict pseudocontractions.

For solving the equilibrium problem, let us give the following assumptions for the bifunction $F$ and the set $C$ :

(A1) $F(x, x)=0$ for all $x \in C$;

(A2) $F$ is monotone, i.e., $F(x, y)+F(y, x) \leq 0$, for all $x, y \in C$;

(A3) for each $x, y, z \in C$, limsup $\sup _{t \rightarrow 0} F(t z+(1-t) x, y) \leq F(x, y)$;

(A4) for each $x \in C, y \mapsto F(x, y)$ is convex and lower semicontinuous.

Lemma 2.2 ([16]) Let $C$ be a nonempty closed and convex subset of a real Hilbert space $H$. For any $x, y, z \in H$ and given also a real number $a \in \mathbb{R}$, the set

$$
\left\{v \in C:\|y-v\|^{2} \leq\|x-v\|^{2}+\langle z, v\rangle+a\right\}
$$

is closed and convex.

Lemma 2.3 ([17]) Let $C$ be a nonempty closed and convex subset of a real Hilbert space $H$. Let $F: C \times C \rightarrow \mathbb{R}$ satisfy(A1)-(A4), and let $r>0$ and $x \in H$. Then there exists $z \in C$ such that

$$
F(z, y)+\frac{1}{r}\langle y-z, z-x\rangle \geq 0, \quad \forall y \in C .
$$

Lemma 2.4 ([18]) Assume that $F: C \times C \rightarrow \mathbb{R}$ satisfies (A1)-(A4). For $r>0$ and $x \in H$, define a mapping $T_{r}: H \rightarrow C$ as follows:

$$
T_{r}(x)=\left\{z \in C: F(z, y)+\frac{1}{r}\langle y-z, z-x\rangle \geq 0, \forall y \in C\right\} .
$$

Then the following hold:

(1) $T_{r}$ is single-valued; 
(2) $T_{r}$ is firmly nonexpansive, i.e., for any $x, y \in H$,

$$
\left\|T_{r} x-T_{r} y\right\|^{2} \leq\left\langle T_{r} x-T_{r} y, x-y\right\rangle
$$

(3) $F\left(T_{r}\right)=\mathrm{EP}(F)$; and

(4) $\mathrm{EP}(F)$ is closed and convex.

Lemma $2.5([7,19])$ Let $H$ be a real Hilbert space. Then the following identities hold:

(i) $\|x-y\|^{2}=\|x\|^{2}-\|y\|^{2}-2\langle x-y, y\rangle, \forall x, y \in H$.

(ii) $\|t x+(1-t) y\|^{2}=t\|x\|^{2}+(1-t)\|y\|^{2}-t(1-t)\|x-y\|^{2}, \forall t \in[0,1], \forall x, y \in H$.

(iii) $\|x+y\|^{2}=\|x\|^{2}+2\langle y, x+y\rangle$.

Lemma 2.6 ([4]) Let $C$ be a nonempty closed and convex subset of a real Hilbert space $H$, and $S: C \rightarrow C$ be a uniformly L-Lipschitz continuous and asymptotically $\lambda$-strict pseudocontraction in the intermediate sense. Then $F(S)$ is closed and convex.

Lemma 2.7 ([4]) Let $C$ be a nonempty closed and convex subset of a real Hilbert space $H$ and $S: C \rightarrow C$ be a uniformly L-Lipschitz continuous and asymptotically $\lambda$-strict pseudocontraction in the intermediate sense. Then the mapping $I-S$ is demiclosed at zero, that is, if the sequence $\left\{x_{n}\right\}$ in $C$ is such that $x_{n} \rightarrow \bar{x}$ and $x_{n}-S x_{n} \rightarrow 0$, then $\bar{x} \in F(S)$.

Lemma 2.8 ([20]) Let $C$ be a nonempty closed and convex subset of a real Hilbert space $H$. Let $\left\{x_{n}\right\}$ be a sequence in $H$ and $u \in H$, and let $q=P_{C} u$. Suppose that $\left\{x_{n}\right\}$ is such that $\omega_{n}\left(x_{n}\right) \subset C$ and satisfies the condition

$$
\left\|x_{n}-u\right\| \leq\|u-q\|, \quad \forall n \in \mathbb{N} .
$$

Then $x_{n} \rightarrow q$.

Lemma 2.9 ([4]) Let $C$ be a nonempty closed and convex subset of a real Hilbert space $H$. Let $S: C \rightarrow C$ be an asymptotically $\lambda$-strict pseudocontractive mapping in the intermediate sense with the sequence $\gamma_{n}$. Then

$$
\left\|S^{n} x-S^{n} y\right\| \leq \frac{1}{1-\lambda}\left(\lambda\|x-y\|+\sqrt{\left(1+(1-\lambda) \gamma_{n}\right)\|x-y\|^{2}+(1-\lambda) c_{n}}\right)
$$

for all $x, y \in C$ and $n \in \mathbb{N}$.

\section{Main results}

In this section, we prove a strong convergence theorem which solves the problem of finding a common solution of a system of equilibrium problems and a common solution of fixed point problems in Hilbert spaces.

Theorem 3.1 Let $C$ be a nonempty closed and convex subset of a real Hilbert space H. Let $M \geq 1$ be a positive integer. Let $\left\{F_{m}\right\}_{m=1}^{M}: C \times C \rightarrow \mathbb{R}$ be a bifunction satisfying(A1)-(A4). Let $\left\{S_{i}\right\}_{i=1}^{N}: C \rightarrow C$ be a uniformly Lipschitz continuous and asymptotically $\lambda_{i}$-strict pseudocontractive mapping in the intermediate sense for some $0 \leq \lambda_{i}<1$ with the sequences $\left\{c_{n, i}\right\} \subset[0, \infty)$ such that $\lim _{n \rightarrow \infty} c_{n, i}=0$ and $\left\{d_{n, i}\right\} \subset[0, \infty)$ such that $\lim _{n \rightarrow \infty} d_{n, i}=0$. Let 
$\lambda=\max \left\{\lambda_{i}: 1 \leq i \leq N\right\}, c_{n}=\max \left\{c_{n, i}: 1 \leq i \leq N\right\}$ and $d_{n}=\max \left\{d_{n, i}: 1 \leq i \leq N\right\}$. Assume that $\Omega:=\left(\bigcap_{m=1}^{M} \operatorname{SEP}\left(F_{m}\right)\right) \cap\left(\bigcap_{i=1}^{N} F\left(S_{i}\right)\right)$ is nonempty and bounded. Let $\left\{\alpha_{n}\right\},\left\{\beta_{n}\right\}$ be sequences in $[0,1]$ such that $0<a \leq \alpha_{n} \leq 1,0<b \leq \beta_{n} \leq 1-\lambda, a, b \in \mathbb{R}, \forall n \in \mathbb{N}$ and $\left\{r_{m, n}\right\}$ be a sequence in $(0, \infty)$ such that $\lim _{n \rightarrow \infty} r_{m, n}>0$.

Let $\left\{x_{n}\right\}$ be a sequence generated by the following algorithm:

$$
\left\{\begin{array}{l}
x_{1} \in C \text { chosen arbitrarily, } \\
C_{1}=H, \\
u_{n}=T_{r_{M, n}}^{F_{M}} T_{r_{M-1, n}}^{F_{M-1}} \cdots T_{r_{2, n}}^{F_{2}} T_{r_{1, n}}^{F_{1}} x_{n}, \\
z_{n}=\left(1-\beta_{n}\right) u_{n}+\beta_{n} S_{i(n)}^{h(n)} u_{n}, \\
y_{n}=\left(1-\alpha_{n}\right) u_{n}+\alpha_{n} z_{n}, \\
C_{n+1}=\left\{w \in C_{n}:\left\|y_{n}-w\right\|^{2} \leq\left\|x_{n}-w\right\|^{2}+\theta_{n}\right\}, \\
x_{n+1}=P_{C_{n+1}}\left(x_{1}\right), \quad \forall n \in \mathbb{N},
\end{array}\right.
$$

where $\theta_{n}=c_{h(n)}+d_{h(n)} \rho_{n}^{2} \rightarrow 0$, as $n \rightarrow \infty$ and $\rho_{n}=\sup \left\{\left\|x_{n}-w\right\|: w \in \Omega\right\}<\infty$ and $n=$ $(h(n)-1) N+i(n)$, where $i(n) \in\{1,2,3, \ldots, N\}$. Then $\left\{x_{n}\right\}$ converges strongly to some point $p^{*}$, where $p^{*}=P_{\Omega}\left(x_{1}\right)$.

Proof The proof is split into seven steps.

Step 1. We will show that $P_{\Omega}$ is well defined.

From Lemma 2.4, we get $\bigcap_{m=1}^{M} \operatorname{SEP}\left(F_{m}\right)$ is closed and convex. From the assumption of $\left\{S_{i}\right\}_{i=1}^{N}$ and Lemma 2.6, it follows that $\bigcap_{i=1}^{N} F\left(S_{i}\right)$ is closed and convex.

Therefore, $\Omega:=\left(\bigcap_{m=1}^{M} \operatorname{SEP}\left(F_{m}\right)\right) \cap\left(\bigcap_{i=1}^{N} F\left(S_{i}\right)\right)$ is closed and convex. Hence, $P_{\Omega}$ is well defined.

Step 2. We will show that $C_{n}$ is closed and convex for each $n \geq 1$.

By the assumption of $C_{n+1}$, it is easy to see that $C_{n}$ is closed for each $n \geq 1$. We only show that $C_{n}$ is convex for each $n \geq 1$.

Note that $C_{1}=H$ is convex. Suppose that $C_{k}$ is convex for some $k \geq 1$. Next, we show that $C_{k+1}$ is convex for the same $k$. For each $w \in C_{k}$, we see that

$$
\left\|y_{k}-w\right\|^{2} \leq\left\|x_{k}-w\right\|^{2}+\theta_{k}
$$

is equivalent to

$$
2\left\langle x_{k}-y_{k}, w\right\rangle \leq\left\|x_{k}\right\|^{2}-\left\|y_{k}\right\|^{2}+\theta_{k} .
$$

Taking $w_{1}$ and $w_{2}$ in $C_{k+1}$ and putting $\bar{w}=t w_{1}+(1-t) w_{2}$, it follows that $w_{1}, w_{2} \in C_{k}$, and so

$$
2\left\langle x_{k}-y_{k}, w_{1}\right\rangle \leq\left\|x_{k}\right\|^{2}-\left\|y_{k}\right\|^{2}+\theta_{k}
$$

and

$$
2\left\langle x_{k}-y_{k}, w_{2}\right\rangle \leq\left\|x_{k}\right\|^{2}-\left\|y_{k}\right\|^{2}+\theta_{k} .
$$

Combining (3.3) with (3.4), we obtain that

$$
2\left\langle x_{k}-y_{k}, \bar{w}\right\rangle \leq\left\|x_{k}\right\|^{2}-\left\|y_{k}\right\|^{2}+\theta_{k} .
$$


That is,

$$
\left\|y_{k}-\bar{w}\right\|^{2} \leq\left\|x_{k}-\bar{w}\right\|^{2}+\theta_{k}
$$

In view of the convexity of $C_{k}$, we see that $\bar{w} \in C_{k}$. This implies that $\bar{w} \in C_{k+1}$. Therefore, $C_{k+1}$ is convex. Hence, $C_{n}$ is closed and convex for each $n \geq 1$.

Step 3. We will show that $\Omega \subset C_{n}$ for each $n \geq 1$.

Put $\Theta_{n}^{m}:=T_{r_{m, n}}^{F_{m}} T_{r_{m-1, n}}^{F_{m-1}} \cdots T_{r_{2, n}}^{F_{2}} T_{r_{1, n}}^{F_{1}} x_{n}$ for every $m \in\{1,2,3, \ldots, M\}$ and $\Theta_{n}^{0}=I$ for all $n \in \mathbb{N}$. Therefore, $u_{n}=\Theta_{n}^{M} x_{n}$. It is obvious that $\Omega \subset C_{1}=H$. Suppose that $\Omega \subset C_{k}$ for some $k \geq 1$.

Next, we show that $\Omega \subset C_{k+1}$ for the same $k$. Taking $p \in \Omega$ and for each $m \in$ $\{1,2,3, \ldots, M\}$, we see that $T_{r_{m, n}}^{F_{m}}$ is nonexpansive and $T_{r_{m, n}}^{F_{m}} p=p$. We note that

$$
\left\|u_{n}-p\right\|=\left\|\Theta_{n}^{M} x_{n}-\Theta_{n}^{M} p\right\| \leq\left\|x_{n}-p\right\|, \quad \forall n \in \mathbb{N} .
$$

We observe that

$$
\begin{aligned}
\left\|z_{n}-p\right\|^{2}= & \left\|\left(1-\beta_{n}\right)\left(u_{n}-p\right)+\beta_{n}\left(S_{i(n)}^{h(n)}\left(u_{n}-p\right)\right)\right\|^{2} \\
= & \left(1-\beta_{n}\right)\left\|u_{n}-p\right\|^{2}+\beta_{n}\left\|S_{i(n)}^{h(n)} u_{n}-p\right\|^{2}-\beta_{n}\left(1-\beta_{n}\right)\left\|S_{i(n)}^{h(n)} u_{n}-u_{n}\right\|^{2} \\
\leq & \left(1-\beta_{n}\right)\left\|u_{n}-p\right\|^{2}+\beta_{n}\left[\left(1+d_{h(n)}\right)\left\|u_{n}-p\right\|^{2}+\lambda\left\|S_{i(n)}^{h(n)} u_{n}-u_{n}\right\|^{2}+c_{h(n)}\right] \\
& -\beta_{n}\left(1-\beta_{n}\right)\left\|S_{i(n)}^{h(n)} u_{n}-u_{n}\right\|^{2} \\
\leq & \left(1+d_{h(n)}\right)\left\|u_{n}-p\right\|^{2}-\beta_{n}\left(1-\beta_{n}-\lambda\right)\left\|S_{i(n)}^{h(n)} u_{n}-u_{n}\right\|^{2}+\beta_{n} c_{h(n)} \\
\leq & \left(1+d_{h(n)}\right)\left\|u_{n}-p\right\|^{2}+\beta_{n} c_{h(n)} .
\end{aligned}
$$

By virtue of convexity of $\|\cdot\|^{2}$, one has

$$
\begin{aligned}
\left\|y_{n}-p\right\|^{2} & =\left\|\left(1-\alpha_{n}\right)\left(u_{n}-p\right)+\alpha_{n}\left(z_{n}-p\right)\right\|^{2} \\
& \leq\left(1-\alpha_{n}\right)\left\|u_{n}-p\right\|^{2}+\alpha_{n}\left\|z_{n}-p\right\|^{2} .
\end{aligned}
$$

Substituting (3.5) and (3.6) into (3.7), we obtain

$$
\begin{aligned}
\left\|y_{n}-p\right\|^{2} & \leq\left(1-\alpha_{n}\right)\left\|u_{n}-p\right\|^{2}+\alpha_{n}\left\|z_{n}-p\right\|^{2} \\
& \leq\left(1-\alpha_{n}\right)\left\|u_{n}-p\right\|^{2}+\alpha_{n}\left[\left(1+d_{h(n)}\right)\left\|u_{n}-p\right\|^{2}+\beta_{n} c_{h(n)}\right] \\
& \leq\left\|u_{n}-p\right\|^{2}+d_{h(n)}\left\|u_{n}-p\right\|^{2}+\beta_{n} c_{h(n)} \\
& \leq\left\|u_{n}-p\right\|^{2}+d_{h(n)}\left\|x_{n}-p\right\|^{2}+c_{h(n)} \\
& =\left\|u_{n}-p\right\|^{2}+\theta_{n} \\
& \leq\left\|x_{n}-p\right\|^{2}+\theta_{n} .
\end{aligned}
$$

Therefore, $p \in C_{k+1}$, and so $\Omega \subset C_{n}$ for each $n \geq 1$. Hence, $\left\{x_{n}\right\}$ is well defined.

Step 4 . We will show that $\left\{x_{n}\right\}$ is bounded.

Since $\Omega$ is a nonempty closed and convex subset of $H$, there exists a unique $q \in \Omega$ such that $q=P_{\Omega} x_{1}$. By the assumption, we have $x_{n}=P_{C_{n}} x_{1}$ for any $q \in \Omega \subset C_{n}$. Then

$$
\left\|x_{n}-x_{1}\right\| \leq\left\|q-x_{1}\right\|=\left\|P_{\Omega} x_{1}-x_{1}\right\|
$$


This implies that $\left\{x_{n}\right\}$ is bounded. Therefore, $\left\{u_{n}\right\},\left\{z_{n}\right\}$, and $\left\{y_{n}\right\}$ are also bounded.

Step 5. We will show that $\left\|u_{n}-S_{i} u_{n}\right\| \rightarrow 0$ and $\left\|x_{n}-S_{i} x_{n}\right\| \rightarrow 0$ as $n \rightarrow \infty, \forall i \in$ $\{1,2,3, \ldots, N\}$.

Since $x_{n}=P_{C_{n}} x_{1}$ and $x_{n}=P_{C_{n}} x_{1} \in C_{n+1} \subset C_{n}$, we have

$$
\begin{aligned}
0 & \leq\left\langle x_{1}-x_{n}, x_{n}-x_{n+1}\right\rangle \\
& =\left\langle x_{1}-x_{n}, x_{n}-x_{1}+x_{1}-x_{n+1}\right\rangle \\
& \leq-\left\|x_{1}-x_{n}\right\|^{2}+\left\|x_{1}-x_{n}\right\|\left\|x_{1}-x_{n+1}\right\| .
\end{aligned}
$$

Therefore, $\left\|x_{1}-x_{n}\right\|^{2} \leq\left\|x_{1}-x_{n}\right\|\left\|x_{1}-x_{n+1}\right\|$, and so

$$
\left\|x_{n}-x_{1}\right\|=\left\|x_{1}-x_{n}\right\| \leq\left\|x_{1}-x_{n+1}\right\| .
$$

Thus, the sequence $\left\{\left\|x_{n}-x_{1}\right\|\right\}$ is nondecreasing. Since $\left\{x_{n}\right\}$ is bounded, $\lim _{n \rightarrow \infty}\left\|x_{n}-x_{1}\right\|$ exists. On the other hand, from (3.10), we have

$$
\begin{aligned}
\left\|x_{n}-x_{n+1}\right\|^{2} & =\left\|x_{n}-x_{1}+x_{1}-x_{n+1}\right\|^{2} \\
& =\left\|x_{n}-x_{1}\right\|^{2}+2\left\langle x_{n}-x_{1}, x_{1}-x_{n+1}\right\rangle+\left\|x_{1}-x_{n+1}\right\|^{2} \\
& =\left\|x_{n}-x_{1}\right\|^{2}+2\left\langle x_{n}-x_{1}, x_{1}-x_{n}+x_{n}-x_{n+1}\right\rangle+\left\|x_{1}-x_{n+1}\right\|^{2} \\
& =\left\|x_{n}-x_{1}\right\|^{2}-2\left\|x_{n}-x_{1}\right\|^{2}+2\left\langle x_{n}-x_{1}, x_{n}-x_{n+1}\right\rangle+\left\|x_{1}-x_{n+1}\right\|^{2} \\
& \leq\left\|x_{1}-x_{n+1}\right\|^{2}-\left\|x_{n}-x_{1}\right\|^{2} .
\end{aligned}
$$

The fact that $\lim _{n \rightarrow \infty}\left\|x_{n}-x_{1}\right\|$ exists implies that

$$
\lim _{n \rightarrow \infty}\left\|x_{n}-x_{n+1}\right\|=0
$$

It is easy to see that

$$
\lim _{n \rightarrow \infty}\left\|x_{n}-x_{n+i}\right\|=0, \quad \forall i=1,2,3, \ldots, N .
$$

Since $x_{n+1}=P_{C_{n+1}} x_{1} \in C_{n+1}$, we have

$$
\left\|y_{n}-x_{n+1}\right\|^{2} \leq\left\|x_{n}-x_{n+1}\right\|^{2}+\theta_{n} .
$$

It follows that

$$
\begin{aligned}
\left\|y_{n}-x_{n}\right\|^{2} & =\left\|y_{n}-x_{n+1}+x_{n+1}-x_{n}\right\|^{2} \\
& =\left\|y_{n}-x_{n+1}\right\|^{2}+\left\|x_{n+1}-x_{n}\right\|^{2}+2\left\langle y_{n}-x_{n+1}, x_{n+1}-x_{n}\right\rangle \\
& \leq\left\|x_{n}-x_{n+1}\right\|^{2}+\theta_{n}+\left\|x_{n+1}-x_{n}\right\|^{2}+2\left\langle y_{n}-x_{n+1}, x_{n+1}-x_{n}\right\rangle \\
& \leq 2\left\|x_{n+1}-x_{n}\right\|^{2}+2\left\|y_{n}-x_{n+1}\right\|\left\|x_{n+1}-x_{n}\right\|+\theta_{n} .
\end{aligned}
$$

Since $\theta_{n} \rightarrow 0$ as $n \rightarrow \infty$ and from (3.13), we obtain

$$
\lim _{n \rightarrow \infty}\left\|x_{n}-y_{n}\right\|=0 .
$$


For each $p \in \Omega$, it follows from the firmly nonexpansive $T_{r_{m, n}}^{F_{m}}$ that for each $m \in\{1,2,3$, $\ldots, M\}$, we have

$$
\begin{aligned}
\left\|\Theta_{n}^{m} x_{n}-p\right\|^{2}= & \left\|T_{r_{m, n}}^{F_{m}} \Theta_{n}^{m-1} x_{n}-T_{r_{m, n}}^{F_{m}} p\right\|^{2} \\
\leq & \left\langle\Theta_{n}^{m} x_{n}-p, \Theta_{n}^{m-1} x_{n}-p\right\rangle \\
= & \frac{1}{2}\left(\left\|\Theta_{n}^{m} x_{n}-p\right\|^{2}+\left\|\Theta_{n}^{m-1} x_{n}-p\right\|^{2}-\left\|\Theta_{n}^{m} x_{n}-\Theta_{n}^{m-1} x_{n}\right\|^{2}\right), \\
& \quad \text { for all } 1 \leq m \leq M .
\end{aligned}
$$

Thus, we get

$$
\left\|\Theta_{n}^{m} x_{n}-p\right\|^{2} \leq\left\|\Theta_{n}^{m-1} x_{n}-p\right\|^{2}-\left\|\Theta_{n}^{m} x_{n}-\Theta_{n}^{m-1} x_{n}\right\|^{2}, \quad \text { for all } 1 \leq m \leq M
$$

This implies that for each $m \in\{1,2,3, \ldots, M\}$,

$$
\begin{aligned}
\left\|\Theta_{n}^{m} x_{n}-p\right\|^{2} \leq & \left\|\Theta_{n}^{0} x_{n}-p\right\|^{2}-\left\|\Theta_{n}^{m} x_{n}-\Theta_{n}^{m-1} x_{n}\right\|^{2}-\left\|\Theta_{n}^{m-1} x_{n}-\Theta_{n}^{m-2} x_{n}\right\|^{2} \\
& -\cdots-\left\|\Theta_{n}^{2} x_{n}-\Theta_{n}^{1} x_{n}\right\|^{2}-\left\|\Theta_{n}^{1} x_{n}-\Theta_{n}^{0} x_{n}\right\|^{2} \\
\leq & \left\|x_{n}-p\right\|^{2}-\left\|\Theta_{n}^{m} x_{n}-\Theta_{n}^{m-1} x_{n}\right\|^{2} .
\end{aligned}
$$

Therefore, by the convexity of $\|\cdot\|^{2}$ and (3.8) and the nonexpansivity of $T_{r_{m, n}}^{F_{m}}$, we get

$$
\begin{aligned}
\left\|y_{n}-p\right\|^{2} & \leq\left\|u_{n}-p\right\|^{2}+\theta_{n} \\
& =\left\|\Theta_{n}^{M} x_{n}-\Theta_{n}^{M} p\right\|^{2}+\theta_{n} \\
& \leq\left\|\Theta_{n}^{m} x_{n}-p\right\|^{2}+\theta_{n} \\
& \leq\left\|x_{n}-p\right\|^{2}-\left\|\Theta_{n}^{m} x_{n}-\Theta_{n}^{m-1} x_{n}\right\|^{2}+\theta_{n} .
\end{aligned}
$$

It follows that

$$
\begin{aligned}
\left\|\Theta_{n}^{m} x_{n}-\Theta_{n}^{m-1} x_{n}\right\|^{2} & \leq\left\|x_{n}-p\right\|^{2}-\left\|y_{n}-p\right\|^{2}+\theta_{n} \\
& \leq\left\|x_{n}-y_{n}\right\|\left(\left\|x_{n}-p\right\|+\left\|y_{n}-p\right\|\right)+\theta_{n} .
\end{aligned}
$$

From (3.15) and (3.17), we obtain

$$
\lim _{n \rightarrow \infty}\left\|\Theta_{n}^{m} x_{n}-\Theta_{n}^{m-1} x_{n}\right\|=0, \quad \forall m \in\{1,2,3, \ldots, M\}
$$

Then we have

$$
\begin{aligned}
\left\|u_{n}-x_{n}\right\| \leq & \left\|u_{n}-\Theta_{n}^{M-1} x_{n}\right\|+\left\|\Theta_{n}^{M-1} x_{n}-\Theta_{n}^{M-2} x_{n}\right\| \\
& +\cdots+\left\|\Theta_{n}^{1} x_{n}-\Theta_{n}^{0} x_{n}\right\| \rightarrow 0, \quad \text { as } n \rightarrow \infty
\end{aligned}
$$

Therefore,

$$
\lim _{n \rightarrow \infty}\left\|u_{n}-x_{n}\right\|=0 .
$$


From (3.13) and (3.19), we get

$$
\left\|u_{n+1}-u_{n}\right\| \leq\left\|u_{n+1}-x_{n+1}\right\|+\left\|x_{n+1}-x_{n}\right\|+\left\|x_{n}-u_{n}\right\| \rightarrow 0, \quad \text { as } n \rightarrow \infty
$$

It follows that

$$
\lim _{n \rightarrow \infty}\left\|u_{n+i}-u_{n}\right\|=0, \quad \forall i \in\{1,2,3, \ldots, N\}
$$

Since for any positive integer $n \geq N$, we can write $n=(h(n)-1) N+i(n)$, where $i(n) \in$ $\{1,2,3, \ldots, N\}$, note that

$$
\begin{aligned}
\left\|u_{n}-S_{n} u_{n}\right\| & \leq\left\|u_{n}-S_{i(n)}^{h(n)} u_{n}\right\|+\left\|S_{i(n)}^{h(n)} u_{n}-S_{n} u_{n}\right\| \\
& =\left\|u_{n}-S_{i(n)}^{h(n)} u_{n}\right\|+\left\|S_{i(n)}^{h(n)} u_{n}-S_{i(n)} u_{n}\right\| .
\end{aligned}
$$

From the conditions $0<a \leq \alpha_{n} \leq 1$ and $0<b \leq \beta_{n} \leq 1-\lambda$, we get

$$
\begin{aligned}
\left\|u_{n}-S_{i(n)}^{h(n)} u_{n}\right\| & =\frac{1}{\beta_{n}}\left\|z_{n}-u_{n}\right\| \\
& =\frac{1}{\alpha_{n} \beta_{n}}\left\|y_{n}-u_{n}\right\| \\
& \leq \frac{1}{a b}\left(\left\|y_{n}-x_{n}\right\|+\left\|x_{n}-u_{n}\right\|\right) .
\end{aligned}
$$

From (3.15) and (3.19), we obtain

$$
\lim _{n \rightarrow \infty}\left\|u_{n}-S_{i(n)}^{h(n)} u_{n}\right\|=0
$$

It is obvious that the relations $h(n)=h(n-N)+1$ and $i(n)=i(n-N)$ hold.

Therefore, we compute

$$
\begin{aligned}
\left\|S_{i(n)}^{h(n)-1} u_{n}-u_{n}\right\| \leq & \left\|S_{i(n)}^{h(n)-1} u_{n}-S_{i(n-N)}^{h(n)-1} u_{n-N+1}\right\|+\left\|S_{i(n-N)}^{h(n)-1} u_{n-N+1}-S_{i(n-N)}^{h(n-N)} u_{n-N}\right\| \\
& +\left\|S_{i(n-N)}^{h(n-N)} u_{n-N}-u_{n-N}\right\|+\left\|u_{n-N}-u_{n-N+1}\right\|+\left\|u_{n-N+1}-u_{n}\right\| \\
= & \left\|S_{i(n)}^{h(n)-1} u_{n}-S_{i(n)}^{h(n)-1} u_{n-N+1}\right\|+\left\|S_{i(n-N)}^{h(n-N)} u_{n-N+1}-S_{i(n-N)}^{h(n-N)} u_{n-N}\right\| \\
& +\left\|S_{i(n-N)}^{h(n-N)} u_{n-N}-u_{n-N}\right\|+\left\|u_{n-N}-u_{n-N+1}\right\|+\left\|u_{n-N+1}-u_{n}\right\| .
\end{aligned}
$$

Applying Lemma 2.9 and (3.21), we get

$$
\lim _{n \rightarrow \infty}\left\|u_{n}-S_{i(n)}^{h(n)-1} u_{n}\right\|=0
$$

From (3.22) and (3.24), it follows that

$$
\lim _{n \rightarrow \infty}\left\|u_{n}-S_{n} u_{n}\right\|=0
$$

Since

$$
\left\|u_{n}-S_{n+i} u_{n}\right\| \leq\left\|u_{n}-u_{n+i}\right\|+\left\|u_{n+i}-S_{n+i} u_{n+i}\right\|+\left\|S_{n+i} u_{n+i}-S_{n+i} u_{n}\right\| \rightarrow 0, \quad \text { as } n \rightarrow \infty
$$


for any $i \in\{1,2,3, \ldots, N\}$, which gives that

$$
\lim _{n \rightarrow \infty}\left\|u_{n}-S_{i} u_{n}\right\|=0, \quad \forall i \in\{1,2,3, \ldots, N\}
$$

Moreover, for each $i \in\{1,2,3, \ldots, N\}$, we obtain

$$
\left\|x_{n}-S_{i} x_{n}\right\| \leq\left\|x_{n}-u_{n}\right\|+\left\|u_{n}-S_{i} u_{n}\right\|+\left\|S_{i} u_{n}-S_{i} x_{n}\right\| \rightarrow 0, \quad \text { as } n \rightarrow \infty .
$$

This implies that

$$
\lim _{n \rightarrow \infty}\left\|x_{n}-S_{i} x_{n}\right\|=0, \quad \forall i \in\{1,2,3, \ldots, N\} .
$$

Step 6. We will show that $p^{*} \in \Omega:=\left(\bigcap_{i=1}^{N} F\left(S_{i}\right)\right) \cap\left(\bigcap_{m=1}^{M} \operatorname{SEP}\left(F_{m}\right)\right)$.

(6.1) We will show that $p^{*} \in \bigcap_{i=1}^{N} F\left(S_{i}\right)$.

We take $p^{*} \in \omega_{w}\left(x_{n}\right)$ and assume that $x_{n_{j}} \rightarrow p^{*}$ for some subsequence $\left\{x_{n_{j}}\right\}$ of $\left\{x_{n}\right\}$.

Note that $S_{i}$ is uniformly Lipschitz continuous and (3.27), we obtain

$$
\lim _{n \rightarrow \infty}\left\|x_{n}-S_{i}^{k} x_{n}\right\|=0, \quad \forall k \in \mathbb{N} .
$$

It follows from Lemma 2.7 that

$$
p^{*} \in \bigcap_{i=1}^{N} F\left(S_{i}\right) \text {. }
$$

(6.2) We will show that $p^{*} \in \bigcap_{m=1}^{M} \operatorname{SEP}\left(F_{m}\right)$.

By Lemma 2.3, for each $m \in\{1,2,3, \ldots, M\}$, we have

$$
F_{m}\left(\Theta_{n}^{m} x_{n}, y\right)+\frac{1}{r_{n}}\left\langle y-\Theta_{n}^{m} x_{n}, \Theta_{n}^{m} x_{n}-\Theta_{n}^{m-1} x_{n}\right\rangle \geq 0, \quad \forall y \in C .
$$

From (A2), we get

$$
\frac{1}{r_{n}}\left\langle y-\Theta_{n}^{m} x_{n}, \Theta_{n}^{m} x_{n}-\Theta_{n}^{m-1} x_{n}\right\rangle \geq F_{m}\left(y, \Theta_{n}^{m} x_{n}\right), \quad \forall y \in C .
$$

Taking $n=n_{j}$, we get

$$
\left\langle y-\Theta_{n_{j}}^{m} x_{n_{j}}, \frac{\Theta_{n_{j}}^{m} x_{n_{j}}-\Theta_{n_{j}}^{m-1} x_{n_{j}}}{r_{n_{j}}}\right\rangle \geq F_{m}\left(y, \Theta_{n_{j}}^{m} x_{n_{j}}\right), \quad \forall y \in C
$$

From (3.18), we obtain that $\Theta_{n_{j}}^{m} x_{n_{j}} \rightarrow p^{*}$ as $j \rightarrow \infty$ for each $m \in\{1,2,3, \ldots, M\}$ (especially $\left.u_{n_{j}}=\Theta_{n_{j}}^{M} x_{n_{j}}\right)$. Considering this together with (3.18) and (A4), we have for each $m \in\{1,2,3, \ldots, M\}$ that

$$
0 \geq F_{m}\left(y, p^{*}\right), \quad \forall y \in C .
$$


For any $0<t \leq 1$ and $y \in C$, we let $y_{t}=t y+(1-t) p^{*}$. Since $y \in C$ and $p^{*} \in C$, we obtain that $y_{t} \in C$, and so $F_{m}\left(y_{t}, p^{*}\right) \leq 0$. It follows that

$$
0=F_{m}\left(y_{t}, y_{t}\right) \leq t F_{m}\left(y_{t}, y\right)+(1-t) F_{m}\left(y_{t}, p^{*}\right) \leq t F_{m}\left(y_{t}, y\right) .
$$

Dividing by $t$, for each $m \in\{1,2,3, \ldots, M\}$, we get

$$
F_{m}\left(y_{t}, y\right) \geq 0, \quad \forall y \in C .
$$

Letting $t \rightarrow 0$, from (A3), we get

$$
F_{m}\left(p^{*}, y\right) \geq 0, \quad \forall y \in C .
$$

Therefore, $p^{*} \in \bigcap_{m=1}^{M} \operatorname{SEP}\left(F_{m}\right)$, and so $p^{*} \in \Omega$.

Step 7. We will show that $\left\{x_{n}\right\}$ converges strongly to $P_{\Omega} x_{1}$.

Set $p^{*}=P_{\Omega}\left(x_{1}\right)$, then

$$
\left\|x_{n+1}-x_{1}\right\| \leq\left\|p^{\prime \prime}-x_{1}\right\|, \quad \forall n \in \mathbb{N} .
$$

Since $\Omega$ is a nonempty closed and convex subset of $H$, there exists a unique $p^{*} \in \Omega$ such that $p^{*}=P_{\Omega}\left(x_{1}\right)$. It follows from Lemma 2.8 that $x_{n} \rightarrow p^{*}$, where $p^{*}=P_{\Omega}\left(x_{1}\right)$. This completes proof.

\section{Deduced theorems}

If we take $M=1$ in Theorem 3.1, then we obtain the following result.

Theorem 4.1 Let $C$ be a nonempty closed and convex subset of a real Hilbert space $H$. Let $M \geq 1$ be a positive integer. Let $F: C \times C \rightarrow \mathbb{R}$ be a bifunction satisfying (A1)-(A4). Let $\left\{S_{i}\right\}_{i=1}^{N}: C \rightarrow C$ be a uniformly Lipschitz continuous and asymptotically $\lambda_{i}$-strict pseudocontractive mapping in the intermediate sense for some $0 \leq \lambda_{i}<1$ with the sequences $\left\{c_{n, i}\right\} \subset[0, \infty)$ such that $\lim _{n \rightarrow \infty} c_{n, i}=0$ and $\left\{d_{n, i}\right\} \subset[0, \infty)$ such that $\lim _{n \rightarrow \infty} d_{n, i}=0$. Let $\lambda=\max \left\{\lambda_{i}: 1 \leq i \leq N\right\}, c_{n}=\max \left\{c_{n, i}: 1 \leq i \leq N\right\}$ and $d_{n}=\max \left\{d_{n, i}: 1 \leq i \leq N\right\}$. Assume that $\Omega:=\operatorname{EP}(F) \cap\left(\bigcap_{i=1}^{N} F\left(S_{i}\right)\right)$ is nonempty and bounded. Let $\left\{\alpha_{n}\right\},\left\{\beta_{n}\right\}$ be sequences in $[0,1]$ such that $0<a \leq \alpha_{n} \leq 1,0<b \leq \beta_{n} \leq 1-\lambda, a, b \in \mathbb{R}, \forall n \in \mathbb{N},\left\{r_{m, n}\right\}$ be a sequence in $(0, \infty)$ such that $\lim _{n \rightarrow \infty} r_{m, n}>0$.

Let $\left\{x_{n}\right\}$ be a sequence generated by the following algorithm:

$$
\left\{\begin{array}{l}
x_{1} \in C \text { chosen arbitrarily, } \\
C_{1}=H, \\
u_{n}=T_{r_{n}}^{F} x_{n}, \\
z_{n}=\left(1-\beta_{n}\right) u_{n}+\beta_{n} S_{i(n)}^{h(n)} u_{n}, \\
y_{n}=\left(1-\alpha_{n}\right) u_{n}+\alpha_{n} z_{n}, \\
C_{n+1}=\left\{w \in C_{n}:\left\|y_{n}-w\right\|^{2} \leq\left\|x_{n}-w\right\|^{2}+\theta_{n}\right\}, \\
x_{n+1}=P_{C_{n+1}}\left(x_{1}\right), \quad \forall n \in \mathbb{N},
\end{array}\right.
$$


where $\theta_{n}=c_{h(n)}+d_{h(n)} \rho_{n}^{2} \rightarrow 0$, as $n \rightarrow \infty$ and $\rho_{n}=\sup \left\{\left\|x_{n}-w\right\|: w \in \Omega\right\}<\infty$ and $n=$ $(h(n)-1) N+i(n)$, where $i(n) \in\{1,2,3, \ldots, N\}$. Then $\left\{x_{n}\right\}$ converges strongly to some point $p^{*}$, where $p^{*}=P_{\Omega}\left(x_{1}\right)$.

Remark 4.2 Theorem 4.1 improves and extends the theorem of Tada and Takahashi [21] and the corollary of Duan and Zhao [7].

If we set $F_{m} \equiv 0$ and $r_{m, n}=1$ for all $m \in\{1,2,3, \ldots, N\}$ in Theorem 3.1, then we obtain the following result.

Theorem 4.3 Let $C$ be a nonempty closed and convex subset of a real Hilbert space $H$. Let $M \geq 1$ be a positive integer. Let $\left\{S_{i}\right\}_{i=1}^{N}: C \rightarrow C$ be a uniformly Lipschitz continuous and asymptotically $\lambda_{i}$-strict pseudocontractive mapping in the intermediate sense for some $0 \leq \lambda_{i}<1$ with the sequences $\left\{c_{n, i}\right\} \subset[0, \infty)$ such that $\lim _{n \rightarrow \infty} c_{n, i}=0$ and $\left\{d_{n, i}\right\} \subset[0, \infty)$ such that $\lim _{n \rightarrow \infty} d_{n, i}=0$. Let $\lambda=\max \left\{\lambda_{i}: 1 \leq i \leq N\right\}, c_{n}=\max \left\{c_{n, i}: 1 \leq i \leq N\right\}$ and $d_{n}=$ $\max \left\{d_{n, i}: 1 \leq i \leq N\right\}$. Assume that $\Omega:=\bigcap_{i=1}^{N} F\left(S_{i}\right)$ is nonempty and bounded. Let $\left\{\alpha_{n}\right\},\left\{\beta_{n}\right\}$ be sequences in $[0,1]$ such that $0<a \leq \alpha_{n} \leq 1,0<b \leq \beta_{n} \leq 1-\lambda, a, b \in \mathbb{R}, \forall n \in \mathbb{N},\left\{r_{m, n}\right\}$ be a sequence in $(0, \infty)$ such that $\lim _{n \rightarrow \infty} r_{m, n}>0$.

Let $\left\{x_{n}\right\}$ be a sequence generated by the following algorithm:

$$
\left\{\begin{array}{l}
x_{1} \in C \text { chosen arbitrarily, } \\
C_{1}=H, \\
z_{n}=\left(1-\beta_{n}\right) x_{n}+\beta_{n} S_{i(n)}^{h(n)} x_{n}, \\
y_{n}=\left(1-\alpha_{n}\right) x_{n}+\alpha_{n} z_{n}, \\
C_{n+1}=\left\{w \in C_{n}:\left\|y_{n}-w\right\|^{2} \leq\left\|x_{n}-w\right\|^{2}+\theta_{n}\right\}, \\
x_{n+1}=P_{C_{n+1}}\left(x_{1}\right), \quad \forall n \in \mathbb{N},
\end{array}\right.
$$

where $\theta_{n}=c_{h(n)}+d_{h(n)} \rho_{n}^{2} \rightarrow 0$, as $n \rightarrow \infty$ and $\rho_{n}=\sup \left\{\left\|x_{n}-w\right\|: w \in \Omega\right\}<\infty$ and $n=$ $(h(n)-1) N+i(n)$, where $i(n) \in\{1,2,3, \ldots, N\}$. Then $\left\{x_{n}\right\}$ converges strongly to some point $p^{*}$, where $p^{*}=P_{\Omega}\left(x_{1}\right)$.

Remark 4.4 Theorem 4.1 improves and extends the theorem of Sahu, Xu, and Yao [4], the theorem of Qin, Cho, Kang, and Shang [3] and the corollary of Duan and Zhao [7].

\section{Numerical examples}

In this section, in order to demonstrate the effectiveness, realization and convergence of algorithm of Theorem 3.1, we consider the following simple example that was presented in reference [4].

Example 5.1 Let $x \in \mathbb{R}$ and $C=[0,1]$. For each $x \in C$, we define

$$
S x= \begin{cases}k x, & \text { if } x \in\left[0, \frac{1}{2}\right] ; \\ 0, & \text { if } x \in\left(\frac{1}{2}, 1\right],\end{cases}
$$

where $0<k<1$. 
It is easy to see that $S: C \rightarrow C$ is discontinuous at $x=\frac{1}{2}$ and $S$ is not Lipschitz continuous. Set $C_{1}=\left[0, \frac{1}{2}\right]$ and $C_{2}=\left(\frac{1}{2}, 1\right]$.

For each $x, y \in C_{1}$, we have

$$
\left|S^{n} x-S^{n} y\right|=k^{n}|x-y| \leq|x-y|, \quad \forall x, y \in C_{1} \text { and } \forall n \in \mathbb{N} \text {. }
$$

For each $x, y \in C_{2}$, we have

$$
\left|S^{n} x-S^{n} y\right|=0 \leq|x-y|, \quad \forall x, y \in C_{2} \text { and } \forall n \in \mathbb{N}
$$

For each $x \in C_{1}$ and $y \in C_{2}$, we have

$$
\begin{aligned}
\left|S^{n} x-S^{n} y\right| & =\left|k^{n} x-0\right| \\
& \leq\left|k^{n}(x-y)+k^{n} y\right| \\
& \leq k^{n}|x-y|+k^{n}|y| \\
& \leq|x-y|+k^{n}, \quad \forall n \in \mathbb{N} .
\end{aligned}
$$

It follows that

$$
\begin{aligned}
\left|S^{n} x-S^{n} y\right|^{2} & \leq\left(|x-y|+k^{n}\right)^{2} \\
& \leq|x-y|^{2}+k\left|x-S^{n} x-\left(y-S^{n} y\right)\right|^{2}+k^{n} K
\end{aligned}
$$

for all $x, y \in C$ and $n \in \mathbb{N}$ and for some $K>0$.

Therefore, $S$ is an asymptotically $k$-strict pseudocontractive mapping in the intermediate sense.

In Theorem 3.1, we set $N=1, F_{m} \equiv 0, \beta_{n}=1-k, \alpha_{n}=\frac{n+1}{2 n}$. We apply it to find the fixed point of $S$ of Example 5.1.

Under the above assumption in Theorem 3.1 is simplified as follows:

$$
\left\{\begin{array}{l}
x_{1} \in H \text { chosen arbitrarily, } \\
C_{1}=H, \\
z_{n}=k x_{n}+(1-k) S^{n} x_{n}, \\
y_{n}=\left(\frac{n-1}{2 n}\right) x_{n}+\left(\frac{n+1}{2 n}\right) z_{n}, \\
C_{n+1}=\left\{w \in C_{n}:\left\|y_{n}-w\right\|^{2} \leq\left\|x_{n}-w\right\|^{2}+\theta_{n}\right\}, \\
x_{n+1}=P_{C_{n+1}} x_{1}, \quad \forall n \in \mathbb{N} .
\end{array}\right.
$$

In fact, in one-dimensional case, $C_{n+1}$ is a closed interval. If we set $\left[a_{n+1}, b_{n+1}\right]:=C_{n+1}$, then the projection point $x_{n+1}$ of $x_{1} \in C$ onto $C_{n+1}$ can be expressed as

$$
x_{n+1}=P_{\Omega}\left(x_{1}\right) \begin{cases}x_{1}, & \text { if } x_{1} \in\left[a_{n+1}, b_{n+1}\right] \\ b_{n+1}, & \text { if } x_{1}>b_{n+1} \\ a_{n+1}, & \text { if } x_{1}<a_{n+1} .\end{cases}
$$


Table 1 The numerical results for an initial guess $x_{1}=0.2,0.5,0.8$

\begin{tabular}{llll}
\hline $\boldsymbol{n}$ (iterative number) & \multicolumn{3}{c}{ Initial guess } \\
\cline { 2 - 4 } & $\mathbf{x}_{\mathbf{1}}=\mathbf{0 . 2}$ & $\boldsymbol{x}_{\mathbf{1}}=\mathbf{0 . 5}$ & $\mathbf{x}_{\mathbf{1}}=\mathbf{0 . 8}$ \\
\hline 10 & 0.1467 & 0.2049 & 0.2105 \\
20 & 0.0163 & 0.0205 & 0.0209 \\
30 & 0.0016 & 0.0019 & 0.0020 \\
40 & $1.5149 \times 10^{-4}$ & $1.8819 \times 10^{-4}$ & $1.9196 \times 10^{-4}$ \\
50 & $1.4889 \times 10^{-5}$ & $1.8494 \times 10^{-5}$ & $1.8864 \times 10^{-5}$ \\
\hline
\end{tabular}

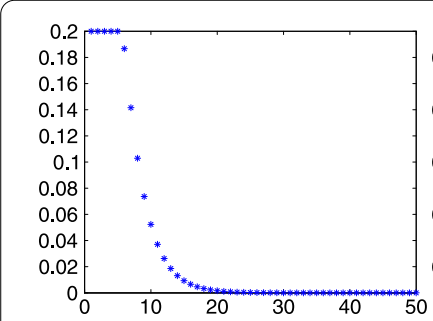

(a) $x_{1}=0.2$

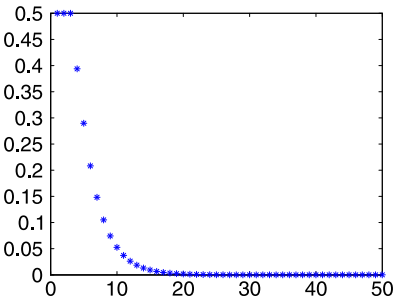

(b) $x_{1}=0.5$

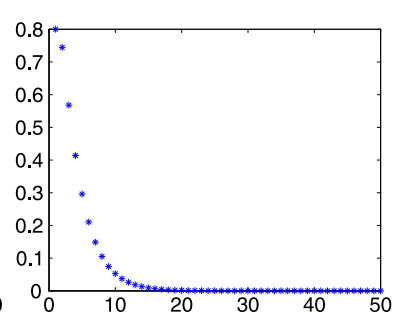

(c) $x_{1}=0.8$

Figure 1 The convergence comparison of different initial values $x_{1}$.

The numerical results for an initial guess $x_{1}=0.2,0.5,0.8$ are shown in Table 1 . From the table, we see that the iterations converge to 0 which is the unique fixed point of $S$. The convergence of each iteration is also shown in Figure 1 for comparison.

Competing interests

The authors declare that they have no competing interests.

Authors' contributions

All authors contributed equally and significantly in this research. All authors read and approved the final manuscript.

\section{Author details}

'Department of Mathematics, Faculty of Science, King Mongkut's University of Technology Thonburi (KMUTT), Bang Mod, Thung Kru, Bangkok, 10140, Thailand. ²Department of Mathematics, Faculty of Education, Bansomdejchaopraya Rajabhat University (BSRU), Thonburi, Bangkok, 10600, Thailand.

\section{Acknowledgements}

This research was supported by the Faculty of Science, KMUTT Research Fund 2553-2554.

Received: 15 June 2012 Accepted: 15 October 2012 Published: 30 October 2012

\section{References}

1. Browder, FE, Petryshyn, WV: Construction of fixed points of nonlinear mappings in Hilbert space. J. Math. Anal. Appl. 20, 197-228 (1967)

2. Liu, F, Nashed, MZ: Regularization of nonlinear ill-posed variational inequalities and convergence rates. Set-Valued Anal. 6, 313-344 (1998)

3. Oin, XL, Cho, YJ, Kang, SM, Shang, M: A hybrid iterative scheme for asymptotically k-strictly pseudocontractions in Hilbert spaces. Nonlinear Anal. 70, 2009 (1902-1911)

4. Sahu, DR, Xu, HK, Yao, JC: Asymptotically strict pseudocontractive mappings in the intermediate sense. Nonlinear Anal. 70, 3502-3511 (2009)

5. Hu, CS, Cai, G: Convergence theorems for equilibrium problems and fixed point problems of a finite family of asymptotically k-strictly pseudocontractive mappings in the intermediate sense. Comput. Math. Appl. 61, 79-93 (2010)

6. Ceng, LC, Ansari, QH, Yao, JC: Strong and weak convergence theorems for asymptotically strict pseudocontractive mappings in the intermediate sense. J. Nonlinear Convex Anal. 11(2), 283-308 (2010)

7. Duan, $\mathrm{P}, \mathrm{Zhao}, \mathrm{J}$ : Strong convergence theorems for system of equilibrium problems and asymptotically strict pseudocontractions in the intermediate sense. Fixed Point Theory Appl. (2011). doi:10.1186/1687-1812-2011-13

8. Ge, C-S: A hybrid algorithm with variable coefficients for asymptotically pseudocontractive mappings in the intermediate sense on unbounded domains. Nonlinear Anal. 75, 2859-2866 (2012) 
9. Geobel, K, Kirk, WA: A fixed point theorem for asymptotically nonexpansive mappings. Proc. Am. Math. Soc. 35, 171-174 (1972)

10. Kirk, WA: Fixed point theorems for non-Lipschitzian mappings of asymptotically nonexpansive type. Isr. J. Math. 17, 339-346 (1974)

11. Bruck, RE, Kuczumow, T, Reich, S: Convergence of iterates of asymptotically nonexpansive mappings in Banach spaces with the uniformly Opial property. Colloq. Math. 65, 169-179 (1993)

12. $\mathrm{Xu}, \mathrm{HK}$ : Existence and convergence for fixed points of mappings of asymptotically nonexpansive type. Nonlinear Anal. 16, 1139-1146 (1991)

13. Kim, TH, Xu, HK: Convergence of the modified Mann's iteration method for asymptotically strict pseudocontractions. Nonlinear Anal. 68, 2828-2836 (2008)

14. Qihou, L: Convergence theorems of the sequence of iterates for asymptotically demicontractive and hemicontractive mappings. Nonlinear Anal. 26, 1835-1842 (1996)

15. Schu, J: Iterative construction of fixed points of asymptotically nonexpansive mappings. J. Math. Anal. Appl. 158, 407-413 (1991)

16. Marino, G, Xu, HK: Weak and strong convergence theorems for strict pseudo-contractions in Hilbert spaces. J. Math. Anal. Appl. 329, 336-346 (2007)

17. Blum, E, Oettli, W: From optimization and variational inequalities to equilibrium problems. Math. Stud. $63,123-145$ (1994)

18. Combettes, PL, Hirstoaga, SA: Equilibrium programming using proximal-like algorithms. Math. Program. 78, 29-41 (1997)

19. Kumam, P, Jaiboon, C: A system of generalized mixed equilibrium problems and fixed point problems for pseudocontractions mappings in Hilbert space. Fixed Point Theory Appl. 2010, Article ID 361512 (2010)

20. Martinez, $\mathrm{CY}, \mathrm{Xu}, \mathrm{HK}$ : Strong convergence theorem of the $\mathrm{CQ}$ method for fixed point processes. Nonlinear Anal. 64, 2400-2411 (2006)

21. Tada, A, Takahashi, W: Weak and strong convergence theorems for a nonexpansive mapping and an equilibrium problem. J. Optim. Theory Appl. 133, 359-370 (2007)

doi:10.1186/1029-242X-2012-252

Cite this article as: Watchararuangwit et al.: A hybrid projection method for solving a common solution of a system of equilibrium problems and fixed point problems for asymptotically strict pseudocontractions in the intermediate sense in Hilbert spaces. Journal of Inequalities and Applications 2012 2012:252.

\section{Submit your manuscript to a SpringerOpen ${ }^{\circ}$ journal and benefit from:}

- Convenient online submission

Rigorous peer review

Immediate publication on acceptance

- Open access: articles freely available online

- High visibility within the field

- Retaining the copyright to your article 